\title{
Textos padronizados em português (BR) para medida da velocidade de leitura - comparação com quatro idiomas europeus
}

\author{
New standardized texts in Brazilian Portuguese to assess reading speed - \\ comparison with four European languages
}

\author{
André Messias ${ }^{1}$ \\ Antonio Augusto Velasco e Cruz ${ }^{2}$ \\ Sonia Jecov Schallenmüller ${ }^{3}$ \\ Susanne Trauzettel-Klosinski ${ }^{4}$
}

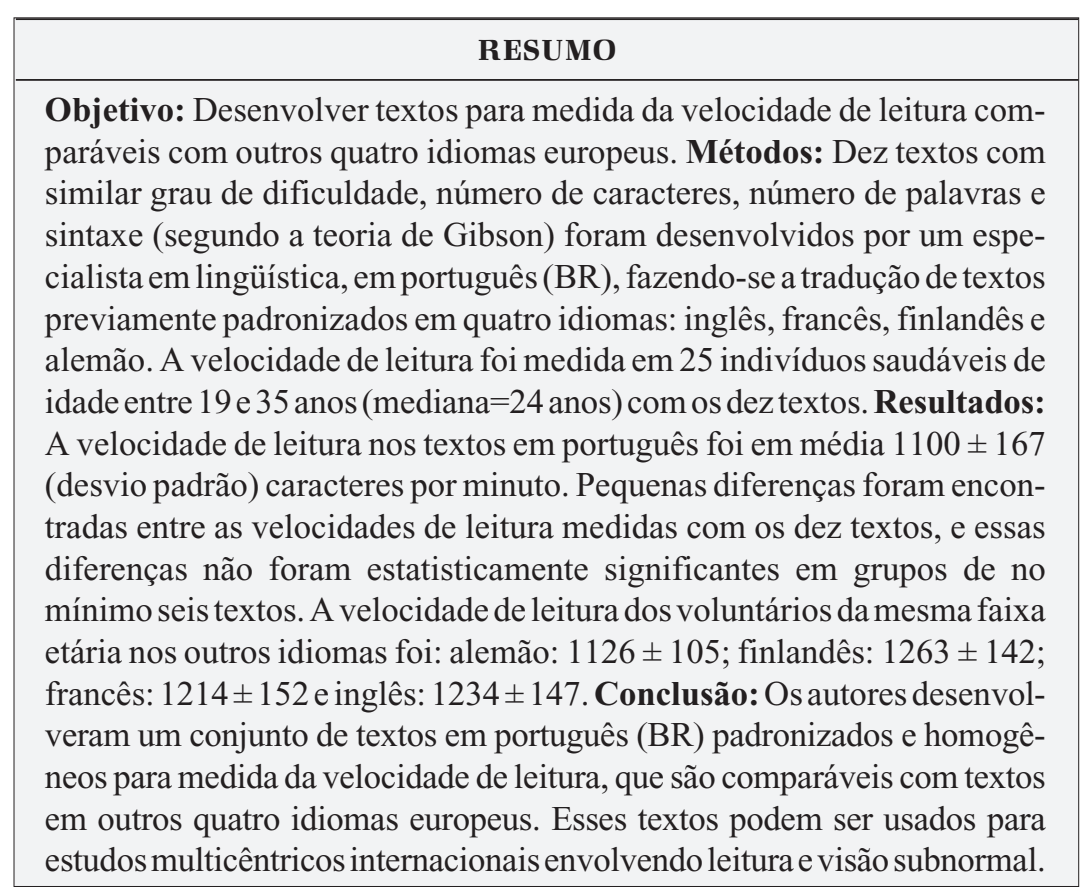

Descritores: Leitura; Movimentos oculares; Baixa visão; Fixação ocular; Acuidade visual; Campos visuais/fisiologia; Testes visuais; Tradução (produto); Vocabulário; Processos mentais; Lingüística; Comparação transcultural

\section{INTRODUÇÃO}

Desde a criação da escrita, a leitura tornou-se uma das mais importantes atividades de formação cultural e integração social do homem. Por definição, leitura é um complexo processo que envolve o sistema sensório, motor e cognitivo, e difere da simples detecção e reconhecimento de letras isoladas (soletrar). Muitos são os fatores que podem influenciar este processo, sendo a integridade do campo visual central um dos principais fatores relacionados a aferência visual ${ }^{(1)}$.

Dificuldade para ler é um dos principais sintomas relatados por pacientes com problemas envolvendo o campo visual central, é citada como uma das principais responsáveis pela piora da qualidade de vida desses pacientes ${ }^{(2)}$, e podem aparecer no decorrer da evolução de certas doenças até mesmo antes da diminuição da acuidade visual central, como por exemplo, em pacientes com doença de Stargardt ${ }^{(3)}$. 
A causa mais freqüente de perda da visão central é a degeneração macular relacionada à idade (DMRI). Com o aumento da população da terceira idade nos países desenvolvidos e em desenvolvimento, a necessidade de métodos para avaliação e programas eficientes de reabilitação visual tem aumentado consideravelmente nas últimas décadas, sem descartar-se o uso de tais programas para pacientes de outras faixas etárias com perdas funcionais semelhantes.

As relações entre a função visual e a estratégia de leitura utilizada por esses pacientes têm sido intensamente estudadas nos últimos anos, e está clara a importância da sua compreensão para a otimização das estratégias de reabilitação ${ }^{(1,4-9)}$. Além disso, habilidade de leitura tem sido incluída como padrão de medida da visão, e tem se mostrado mais sensível do que a medida da acuidade visual na detecção desses problemas ${ }^{(10-12)}$.

A área da retina utilizada no processo de leitura compreende alguns milímetros quadrados, que são desproporcionalmente representados no córtex visual. Os $10^{\circ}$ centrais do campo visual, que correspondem a aproximadamente $2 \%$ do campo total, utilizam mais de $50 \%$ do córtex visual primário ${ }^{(13)}$. Enquanto a acuidade visual, de acordo com a densidade de fotorreceptores, diminui exponencialmente com o aumento da excentricidade na retina. Assim, a acuidade visual, que na fovéola normal é no mínimo igual a 1 minuto de arco, na borda foveal cai para 0,4 , e esse último compreende o mínimo poder resolutivo necessário para o reconhecimento de letras de impressão padronizadas de jornais (Fonte: Times new roman, tamanho: 9 pontos a $25 \mathrm{~cm}$ de distância $)^{(1)}$.

Durante o processo de leitura, uma área relativamente pequena de campo visual é requerida: $4^{\circ}$ no meridiano horizontal e $2^{\circ}$ no vertical, distribuídos simetricamente em torno do ponto de fixação ${ }^{(1)}$. Esta área mínima de visão foi recentemente denominada "visual span" e corresponde, aproximadamente, ao tamanho da fóvea ${ }^{(14)}$. A capacidade de leitura é então determinada, por um lado, pela resolução espacial da retina e, por outro, pela área mínima de percepção visual.

Durante o processo normal de leitura, são captados seqüencialmente grupos de letras. Para cada captura, o sistema oculomotor estabiliza a posição ocular durante um intervalo de tempo da ordem de $150 \mathrm{~ms}$, para que a imagem contendo as letras possa ser visualmente e cognitivamente processada. Tal intervalo é denominado "fixação", e sua duração é também dependente da presença ou não de escotoma central ${ }^{(15)}$.

Entre as sucessivas fixações, são realizados movimentos sacádicos oculares. Estes movimentos são de alta velocidade, são realizados da esquerda para a direita (línguas européias), e têm amplitude aproximada de $4^{\circ}$ de ângulo visual. Ao fim das linhas ocorrem os retornos, que são movimentos de maior amplitude, da direita para a esquerda, realizados para se encontrar o início da próxima linha. Alguns pequenos sacádicos corretores da esquerda para direita podem também ocorrer durante o processo.

Vários testes para medida da habilidade de leitura têm sido desenvolvidos. Entre eles os principais são a tabela de leitura de palavras Bailey-Lovie ${ }^{(16)}$ e a tabela "Minnesota Low Vision Reading Test" (MNREAD) ${ }^{(17)}$, este último recentemente traduzido e adaptado para o português brasileiro ${ }^{(18)}$. Esses testes são essenciais para medida da acuidade de leitura e do aumento necessário para pacientes com baixa visão, porém eles consistem de frases únicas e relativamente curtas, e podem mascarar dificuldades para ler textos longos e com várias linhas.

Considerando a complexidade envolvida na leitura, esta deve ser avaliada em textos contínuos e com várias linhas, pois essas são as condições encontradas em jornais, livros, bulas de remédios, etc. Tais textos devem ser padronizados quanto ao tamanho, dificuldade de compreensão e sintaxe, e freqüência das palavras para que os resultados obtidos possam ser interpretados corretamente.

Recentemente, o grupo europeu AMD-Read (http://www.amdread.net) desenvolveu um conjunto de textos para medida da velocidade de leitura em 4 idiomas europeus: Alemão, Finlandês, Francês e Inglês ${ }^{(19)}$, o objetivo do presente trabalho é traduzir e adaptar esses textos para o português brasileiro e avaliar a performance de indivíduos normais em comparação com os outros quatro idiomas.

\section{MÉTODOS}

\section{Textos}

\section{Conteúdo}

Os dez textos originais foram retirados de uma enciclopédia escrita para crianças entre 9 e 11 anos, e de material de leitura para a sexta série do currículo escolar alemão (equivalente à sexta série da grade brasileira) e posteriormente traduzidos e adaptados para os demais idiomas por especialistas em lingüística de cada país. Todos os textos contêm passagens de interesse geral e de baixa dificuldade de compreensão para assegurar que as habilidades intelectuais não sejam principais fatores responsáveis por possíveis dificuldades na leitura.

\section{Número de caracteres}

Os textos em português possuem em média de $813 \pm 13$ caracteres, enquanto textos nos outros quatro idiomas tiveram em média $830 \pm 2$ caracteres. A razão número de caracteres por palavra nos dez textos dos cinco idiomas foi $6,30 \pm 0,06 \mathrm{em}$ alemão; 7,85 $\pm 0,09$ em finlandês; 6,18 $\pm 0,08$ em francês; $5,43 \pm 0,11$ em inglês e $6,11 \pm 0,09$ em português brasileiro.

\section{Freqüência das palavras e sintaxe}

A freqüência das palavras utilizadas e a sintaxe são fatores determinantes na velocidade de leitura de um texto. Por isso, foram utilizadas somente palavras com freqüência de no mínimo $0.0001 \%$, em frases de no máximo quatro "unidades de integração sintática" segundo a teoria de Gibson ${ }^{(20-21)}$. Este valor é definido como sendo proporcional à distância entre as palavras integradas e o centro semântico das orações.

\section{Layout}

Os dez textos foram impressoss com $98 \%$ de contraste com uma impressora laser comercial (HP LaserJet 4500). A fonte utili- 
zada foi "Times New Roman" de 9 pontos com espaçamento simples entre as linhas para se aproximar ao máximo do padrão usado em jornais. A figura 1 mostra um dos textos em português.

\section{Voluntários}

A população de estudo consistiu de 25 brasileiros, e os resultados foram avaliados juntamente com dados previamente publicados $^{(19)}$ de voluntários ingleses, finlandeses, franceses e alemães. Distribuição das idades e gêneros é mostrada na tabela 1. Não houve diferença estatisticamente significante de idade entre os indivíduos de diferentes nacionalidades $(\mathrm{F}=0,605 ; \mathrm{p}=0,6594)$.

\section{Medida da velocidade de leitura}

Os testes foram realizados com os voluntários sentados confortavelmente em frente a uma bancada de leitura a uma distância fixa de $25 \mathrm{~cm}$ com luminária para garantir boa iluminação.

Os textos foram apresentados em ordem randômica e os voluntários foram orientados a lê-los em voz alta o mais rápido possível sem cometer erros, e se caso erros cometessem não parar a leitura para corrigi-los. O tempo de leitura foi medido com cronômetro comercial e a velocidade de leitura foi calculada dividindo-se o número de caracteres do texto lido pelo tempo gasto. O número de caracteres das palavras não pronunciadas ou lidas erroneamente foram descontadas no total de caracteres no texto.

Ao invés do número de palavras por minuto, número de caracteres lidos por minuto foi unidade usada para comparação

Árvores crescem praticamente em toda parte, exceto no gelo constante, na neve eterna, nos cumes das montanhas altas e no deserto. Se um terreno vazio é abandonado por um período relativamente longo, ali crescem árvores depois de um tempo. Inicialmente, o solo se cobre de plantas rasteiras. Arbustos crescem mais tarde, em cuja sombra morre uma parte das plantas rasteiras que haviam surgido primeiro. Depois de mais algum tempo, árvores começam a crescer. Quando elas ficam maiores, uma parte dos arbustos é atingida por sua sombra e eles morrem. Desse modo, ao passar do tempo, forma-se uma floresta. A maioria das árvores cresce lentamente, algumas delas podem viver muitos anos. Quando as árvores velhas morrem, surgem novas ocupando seu lugar. As florestas são espaços vitais que podem permanecer inalterados por longo tempo.

Figura 1 - Texto em português (BR) das velocidades de leitura, pois o tamanho das palavras varia muito entre os idiomas. Para facilitar compreensão, alguns resultados são mostrados também em palavras por minutos.

\section{Análise estatística}

Two-way análise de variância foi realizada para comparar efeitos dos textos e idiomas na velocidade de leitura. Em seguida, o teste Tukey-HSD foi aplicado para comparar as médias. Foram considerados significativos valores de $p$ menores do que 0,05 . A análise estatística foi realizada com o programa JMP-IN versão 5.1 .

\section{RESULTADOS}

\section{Comparação entre os textos em português}

Houve diferença estatisticamente significante entre as velocidades de leitura medidas com alguns textos (ANOVA: $\mathrm{F}=12,71 ; \mathrm{p}<0,001)$. A máxima diferença encontrada entre a velocidade de leitura medida com os textos em português foi $12 \%$, encontrada comparando-se texto número 4 (lido mais rapidamente) e texto número 1 . A figura 2 mostra a velocidade de leitura de todos os textos em todos os idiomas. A tabela 2 lista as médias e o desvio padrão da velocidade de leitura medida com todos os textos em português e o resultado da análise estatística, onde textos não conectados pela mesma letra apresentaram diferença estatisticamente significante.

Conjuntos de pelo menos seis textos em português podem ser utilizados sem que diferenças entre as velocidades de leitura sejam esperadas.

\section{Comparação entre os idiomas}

As médias das velocidades de leitura em caracteres por minuto foram: Alemão: $1126 \pm 105$; Finlandês: $1263 \pm 142$; Francês: $1214 \pm 152$; Inglês: $1234 \pm 147$; e Português $1100 \pm 167$. Apesar de pequena, houve diferença estatisticamente significante entre as velocidades de leitura entre alguns dos idiomas (Figura 3, Tabela 3), porém com variabilidade das medidas muito semelhantes.

\section{DISCUSSÃO}

A medida objetiva da velocidade de leitura é fundamental na avaliação de pacientes com visão subnormal, principalmen-

\begin{tabular}{|c|c|c|c|c|c|}
\hline \multirow[t]{2}{*}{ Grupo } & & dade (anc & & Núme & duos \\
\hline & Mínima & Máxima & Mediana & Mulheres & Homens \\
\hline Alemão & 18 & 33 & 26 & 15 & 10 \\
\hline Finlandês & 18 & 35 & 26 & 13 & 12 \\
\hline Francês & 20 & 35 & 30 & 10 & 15 \\
\hline Inglês & 19 & 34 & 27 & 12 & 13 \\
\hline Português (BR) & 18 & 35 & 24 & 15 & 10 \\
\hline
\end{tabular}




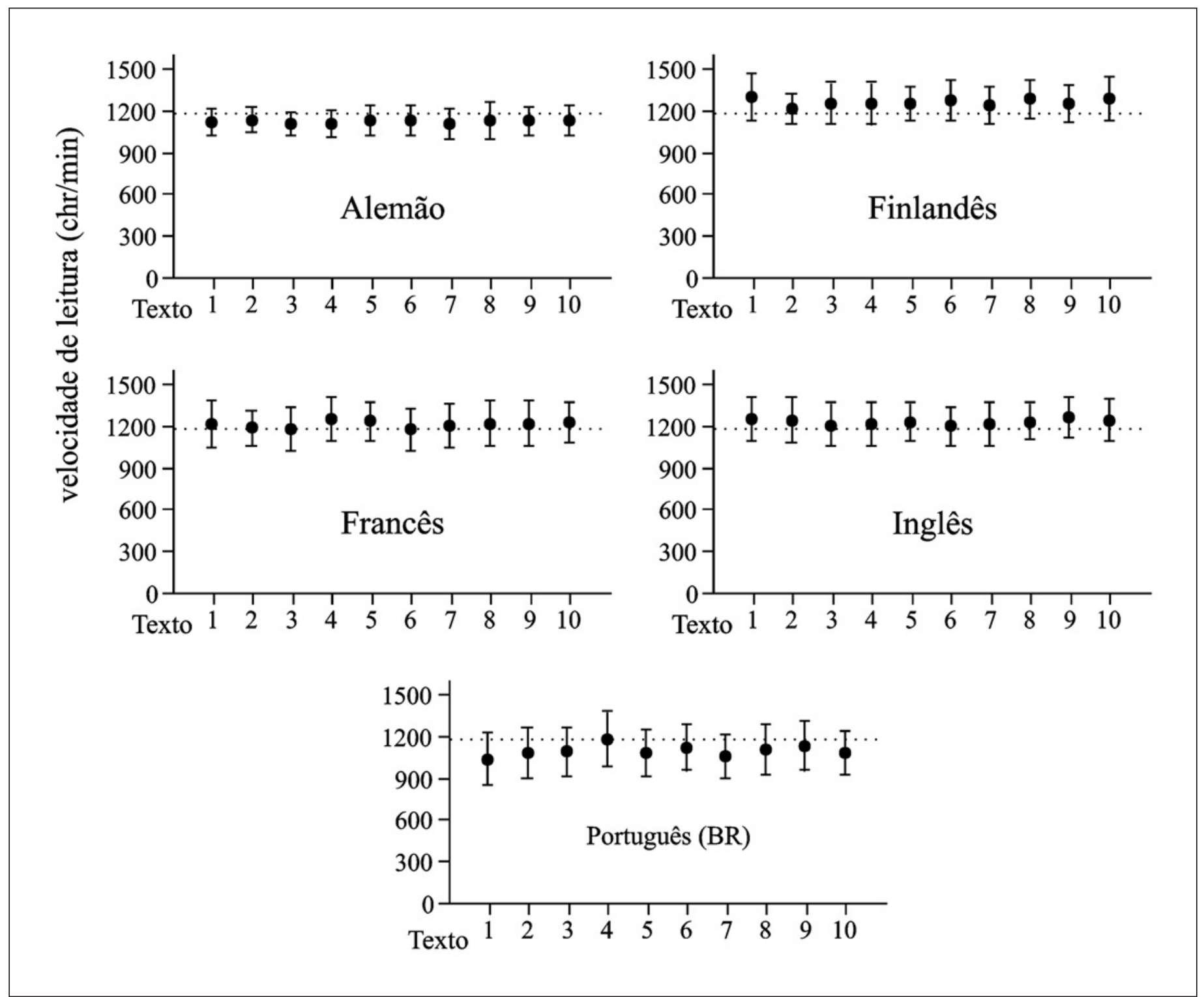

Figura 2 - Pontos representam as médias e as barras verticais \pm 1 desvio padrão das velocidades de leitura em caracteres por minuto de 25 indivíduos com visão normal em todos os textos e em todos os idiomas. A linha tracejada representa a média entre todas as medidas em todos os idiomas $=1187$ caracteres $/$ minuto.

\begin{tabular}{|c|c|c|c|c|c|c|c|c|c|}
\hline Texto & Palavras & Caracteres & $\begin{array}{c}\text { Palavras por minuto } \\
\text { média } \pm \text { DP }\end{array}$ & $\begin{array}{c}\text { Caracteres por minuto } \\
\text { média } \pm \text { DP }\end{array}$ & \multicolumn{5}{|c|}{ Tukey-HSD* } \\
\hline 4 & 131 & 831 & $186 \pm 30$ & $1183 \pm 193$ & A & & & & \\
\hline 9 & 130 & 811 & $182 \pm 29$ & $1137 \pm 183$ & $A$ & B & & & \\
\hline 6 & 123 & 798 & $172 \pm 26$ & $1118 \pm 168$ & & B & C & & \\
\hline 8 & 138 & 798 & $192 \pm 31$ & $1108 \pm 178$ & & B & C & $\mathrm{D}$ & \\
\hline 3 & 132 & 831 & $174 \pm 28$ & $1097 \pm 176$ & & B & C & $\mathrm{D}$ & \\
\hline 2 & 140 & 812 & $187 \pm 31$ & $1085 \pm 182$ & & & C & $\mathrm{D}$ & $E$ \\
\hline 5 & 131 & 810 & $175 \pm 27$ & $1084 \pm 165$ & & & C & $\mathrm{D}$ & $E$ \\
\hline 10 & 128 & 816 & $170 \pm 25$ & $1082 \pm 161$ & & & C & $\mathrm{D}$ & $E$ \\
\hline 7 & 148 & 827 & $191 \pm 28$ & $1065 \pm 159$ & & & & $\mathrm{D}$ & $E$ \\
\hline 1 & 133 & 800 & $173 \pm 31$ & $1040 \pm 185$ & & & & & $E$ \\
\hline
\end{tabular}




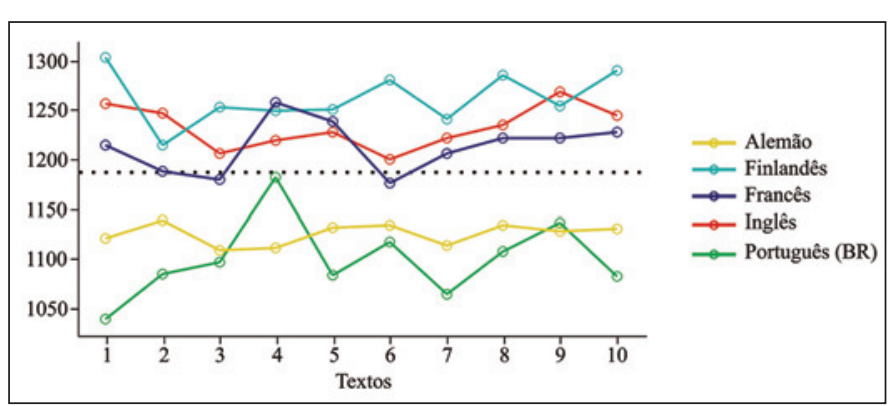

Figura 3 - Pontos representam as médias das velocidades de leitura medidas em cada texto por idioma. A linha tracejada representa a média entre todas as medidas em todos os idiomas = 1187 caracteres/minuto.

\begin{tabular}{|c|c|c|c|}
\hline \multicolumn{3}{|c|}{ Tukey HSD* } & \multirow{2}{*}{$\begin{array}{c}\text { Média } \pm \text { DP } \\
1126 \pm 105\end{array}$} \\
\hline Alemão & & C & \\
\hline Finlandês & A & & $1263 \pm 142$ \\
\hline Francês & & B & $1214 \pm 152$ \\
\hline Inglês & A & B & $1234 \pm 147$ \\
\hline Português (BR) & & C & $1010 \pm 175$ \\
\hline
\end{tabular}

te em estudos com novas terapias para doenças que acometem o campo visual central ${ }^{(10)}$. Para realizá-la, é necessário um conjunto de textos homogêneos quanto ao conteúdo e forma, e que sejam lidos com velocidades comparáveis, para que o pesquisador possa oferecer diferentes textos em diferentes consultas, ou realizar múltiplas medidas.

Os testes previamente descritos para avaliação da leitura apresentam algumas dessas características. As frases que compõem o MNREAD, por exemplo, foram criadas com o mesmo número de caracteres, tem palavras de mesma freqüência e estão disponíveis em vários idiomas. Esse teste pode ser aplicado em diferentes tamanhos de letras e é útil para determinar o quanto texto deve ser aumentado em tamanho (magnification need) que um paciente com baixa visão necessita para ler, todavia é composto por sentenças únicas e curtas, o que impede uma exata avaliação do desempenho de leitura em textos contínuos. Com isso, esses pacientes não precisam, por exemplo, movimentar o sistema de aumento sobre o texto ou encontrar a próxima linha.

Os resultados do presente estudo mostram que a velocidade de leitura dos textos em português de um pouco varia em indivíduos com visão normal. Porém, pequenas diferenças são encontradas quando diferentes textos e idiomas são comparados, de modo que adequada utilização desses textos permite a comparação do desempenho de leitura. É possível formar subgrupos de até seis textos que não apresentam velocidade de leitura estatisticamente significante diferente entre si (por exemplo, tabela 2 - textos marcados com as letras C e D), resultados similares aos encontrados por alguns autores em outros idio$\operatorname{mas}^{(19)}$. Assim, velocidades de leituras medidas com esses sub- grupos de textos poderiam ser diretamente comparadas em futura aplicação clínica. No caso de necessidade de um conjunto com maior número de textos, os dez textos poderiam ser aplicados desde que a velocidade de leitura seja corrigida de acordo com os seus valores normativos.

Em caso de comparações entre a performance em diferentes idiomas, o mais importante é pequena diferença entre as variabilidades (desvio padrão) encontrada em cada um: desvio padrão mínimo encontrado foi de 105 caracteres/minuto (9\% da média) em alemão e o máximo foi de 175 caracteres/ minuto ( $16 \%$ da média) em português; o que mostra que apesar das diferenças estruturais de cada idioma - número médio de caracteres por palavra, colocação dos verbos, construções gramaticais, e diferenças de dificuldade na pronúncia - levarem a uma pequena, porém estatisticamente significante diferença entre as velocidades de leitura em alguns idiomas, a leitura dos textos traduzidos podem ser comparadas se corrigidas com os seus valores normativos.

\section{CONCLUSÃO}

Em suma, um conjunto de textos contínuos de passagens completas foi desenvolvido, os autores os recomendam para medida da velocidade de leitura em avaliações clínicas, principalmente em casos de defeitos no campo visual central em pacientes com baixa visão.

\section{AGRADECIMENTOS}

Os autores agradecem Paula Chiaradia por recrutar os voluntários brasileiros.

\section{ABSTRACT}

Purpose: To develop standardized texts in Brazilian Portuguese to assess reading speed and compare performances among four European languages. Methods: 10 texts were designed by a linguistic expert at the level of a sixth grade reading material (reading ages 10-12 years) and were matched for length and syntactic complexity, according to the syntactic prediction locality theory of Gibson. Normally sighted native speaking volunteers aged 18-35 years read each text aloud at random. Results: The reading speed was on average $1100 \pm 167$ (standard deviation) characters per minute. Only small differences were found between the measured reading speeds of the 10 texts, and these differences were not statistically significant in groups of at least 6 texts. The mean reading speed in age-matched volunteers in Finnish was: $1263 \pm 142$; French: $1214 \pm 152$; English: $1234 \pm 147$; and German: $1126 \pm 105$. Conclusions: The authors developed a set of standardized, homogeneous, and comparable texts in Brazilian Portuguese. These texts will be a valuable tool to measure reading speed for repeated measurements and in international studies in the field of reading and low vision research. 
Keywords: Reading; Eye movements; Vision, low; Fixation, ocular; Visual acuity; Visual fields/physiology; Vision tests; Translations; Vocabulary; Mental processes; Linguistics; Cross-cultural comparison

\section{REFERÊNCIAS}

1. Aulhorn E. [Fixation width and fixation frequency of the contours presented in reading.]. Pflugers Arch. 1953;257(4):318-28.

2. Mitchell J, Bradley C. Quality of life in age-related macular degeneration: a review of the literature. Health Qual Life Outcomes. 2006;4:97.

3. Rohrschneider K, Gluck R, Blankenagel A, Volcker HE. [Fixation behavior in Stargardt disease. Fundus-controlled studies]. Ophthalmologe. 1997;94(9): 624-8. German.

4. Legge GE, Rubin GS, Pelli DG, Schleske MM. Psychophysics of reading-II Low vision. Vision Res. 1985;25(2):253-65.

5. Nilsson UL, Frennesson C, Nilsson SE. Location and stability of a newly established eccentric retinal locus suitable for reading, achieved through training of patients with a dense central scotoma. Optom Vis Sci. 1998;75(12):873-8.

6. Timberlake GT, Peli E, Essock EA, Augliere RA. Reading with a macular scotoma. II. Retinal locus for scanning text. Invest Ophthalmol Vis Sci. 1987; 28(8):1268-74.

7. Trauzettel-Klosinski S, Tornow R. Fixation behavior and reading ability in macular scotoma - assessed by Tübingen manual perimetry and Scanning laser ophthalmoscopy. Neuroophthalmology. 1996;16(3):241-53.

8. Trauzettel-Klosinski S, Reinhard J. The vertical field border in hemianopia and its significance for fixation and reading. Invest Ophthalmol Vis Sci. 1998; 39(11):2177-86.
9. Trauzettel-Klosinski S, Hahn GA. Support for patients loosing sight. Dev Ophthalmol. 2003;37:199-214

10. Fletcher DC, Schuchard RA. Visual function in patients with choroidal neovascularization resulting from age-related macular degeneration: the importance of looking beyond visual acuity. Optom Vis Sci. 2006;83(3):178-89.

11. Crossland MD, Culham LE, Rubin GS. Fixation stability and reading speed in patients with newly developed macular disease. Ophthalmic Physiol Opt. 2004 24(4):327-33

12. Lovie-Kitchin J, Feigl B. Assessment of age-related maculopathy using subjective vision tests. Clin Exp Optom. 2005;88(5):292-303.

13. Horton JC, Hoyt WF. The representation of the visual field in human striate cortex A revision of the classic Holmes map. Arch Ophthalmol. 1991;109(6):816-24.

14. Legge GE, Ahn SJ, Klitz TS, Luebker A. Psychophysics of reading-XVI. The visual span in normal and low vision. Vision Res. 1997;37(14):1999-2010.

15. Bullimore MA, Bailey IL. Reading and eye movements in age-related maculopathy. Optom Vis Sci. 1995;72(2):125-38.

16. Bailey IL, Lovie JE. The design and use of a new near-vision chart. Am J Optom Physiol Opt. 1980;57(6):378-87.

17. Legge GE, Ross JA, Luebker A, LaMay JM. Psychophysics of reading. VIII. The Minnesota Low-Vision Reading Test. Optom Vis Sci. 1989;66(12):843-53.

18. Castro CT, Kallie CS, Salomão SR. [Development and validation of the MNREAD reading acuity chart in Portuguese]. Arq Bras Oftalmol. 2005; 68(6):777-83.

19. Hahn GA, Penka D, Gehrlich C, Messias A, Weismann M, Hyvarinen L, et al. New standardised test for assessing reading performance in four European languages. Br J Ophthalmol. 2006;90(4):480-4.

20. Grodner D, Gibson E, Watson D. The influence of contextual contrast on syntactic processing: evidence for strong-interaction in sentence comprehension. Cognition. 2005;95(3):275-96.

21. Gibson E. Linguistic complexity: locality of syntactic dependencies. Cognition. 1998;68(1):1-76. 